\title{
ZU ROBERT BRUNNE, HANDLYNG SYNNE $9863-66$.
}

(Berichtigung zu Angla 37, 52, anmerkung 2.)

In meiner behandlung von ae. cumcedre 'comother' hatte ich a. a. o. eine stelle aus R. Brunne, "Handling of Synne 986" angezogen, worin angeblich "die eltern ausdrücklich davor gewarnt werden, ihre kinder selbst dem bischof über die taufe zu halten" und "sterilität des kindes als folge solch unbedachten handelns hingestellt" wird. Ich entnahm die stelle der angabe des NED. unter cummer und berichtige zunächst mein versehen Handling of Synne statt Handlyng Synne. Auf versehen des NED. aber beruht die falsche versangabe 986 . Commare kommt nicht in diesem verse, sondern vielmehr in vers 9865 vor und das ganze zitat umfafst die verse 9863-66. Ich habe die betreffende schrift von Robert Brunne erst hier in der ausgabe von Furnivall einsehen können und es hat mich einige zeit gekostet, bevor ich die stelle fand. Doch war es der mühe wert. Denn ich bin nunmehr in der lage festzustellen, dafs es sich in dem zitate nicht um taufpaten handelt. Die verse gehören vielmehr zu einer belehrung über die wichtigkeit des sakraments der konfirmation und von personen, die als paten des kindes bei dieser heiligen handlung unzulässig sind, ist da die rede. Unzulässig aber sind darnach die eignen eltern und blutsverwandten des kindes; diese werden gewarnt, als konfirmationspaten ihrer kinder zu fungieren, weil dies ein gesetzliches hindernis für deren spätere verheiratung ist. Da auch Furnivall den passus nicht recht verstanden zu haben scheint - er überschreibt ihn wenigstens: "Beware of presenting a Friend's Child or a Girl to the 
Bishop" - und da die ganze sache von allgemeinerem interesse ist, so will ich den abschnitt von vers 9861 an vollständig hersetzen:

9861 Anoper poynt here-to longep, pat lewed men nat vndyrstandyp:

"pou man or woman, be nat so wylde",

"To holde to be bysshope pyn owne childe",

9865 "For gyf pou do, pou art commare"

"To hym, pat hyt gat or bare";

"Gyf a frende man holde hyt pore",

"To wedde pat chylde, he may neuer more."

Beachtung verdient der gebrauch von commare v. 9865 . Nach dem NED. müfste man annehmen, es bedeute da ' $a$ godmother in her relationship to the other god-parents of the child, a commother'. Aber diese definition ist viel zu enge für unsere stelle; ganz augenscheinlich umfalst da commare den begriff von godfather sowohl wie godmother, steht also im sinne von co-parent, wie denn auch der begriff von hym im folgenden verse ebenso weit ist: er schliefst sowohl den vater ein, der das kind erzeugte, als die mutter, die es gebar. $A$ frende man v. 9687 ist nicht mit Furnivall als 'freund' im gewöhnlichen sinne des wortes zu fassen, sondern bedeutet hier 'blutsverwandter' und diese stelle wäre im NED. unter friend 3 'a kinsman or near relation' nach dem zitate vom jahre 1300 nachzutragen.

Noch viel auffälliger als der oben nachgewiesene allgemeinere sinn von commare ist der von godmother bei demselben R. Brunne, Handlyng Synne 1695, und er ist da um so merkwürdiger, als im vorhergehenden verse das wort in der eigentlichen bedeutung erscheint:

Also shal pe womman wande (erklärt spare)

To take here godmodrys husbonde.

- Twey godmodrys shul nat wedde, be toon to aske pe touper to bedde.

Es ist bezeichnend, daIs $\mathrm{D}$ für die beiden letzten verse liest:

to commatres schal bou not wedde, pat oon aske pat oper to bedde. 
Weder von dem hier bezeugten commater nimmt das NED. unter cummer notiz, noch verzeichnet es unter godmother den eigentümlichen gebrauch von godmother im sinne von godparent, sponsor. Überhaupt ist, wie ich $\mathrm{zu}$ meiner verwunderung beim durchlesen der schrift beobachtet habe, Robert Brunne's Handlyng Synne bei weitem nicht in dem umfange für belege im NED. ausgenutzt worden, als man wohl hätte erwarten sollen. So wird als erster beleg für das vb. disturble der c. 1330 datierte beleg aus R. Brunne Chron. Wace 1106 aufgeführt, während ein 27 jahre früherer beleg aus desselben autors Handlyng Synne v. 4731 hätte gegeben werden können:

pat he (the minstrel) desturbled pe benesoun

And pe gode mannys deuocyoun.

Desgleichen wird für das verwandte vb. distrouble der c. 1369 datierte beleg aus Chaucer, Dethe Blaunche 524 als erster gegeben, während ein 66 jahre älterer beleg aus Brunne's Handlyng Synne $1214 \mathrm{zu}$ haben war: gyf pou destroblyst here testament. Ebenso vermilst man mit verwunderung unter glittering ppl. a. zwischen den beiden 13 .. und a. 1400-50 datierten belegen, die gleterand, gletirund aufweisen, den 1303 $\mathrm{zu}$ datierenden aus R. Brunne's Handlyng Synne 1407-8 $y$ sagh pere housys of ful ryche atyre / Alle of gletryng golde as fyre, der wichtig ist, weil er schon den übergang von -and zu -ing bezeugt.

Für down-right $4 \mathrm{im}$ sinne von straightway, straight gibt das NED. als ersten den späten beleg aus dem jahre 1647, während aus R. Brunne's Handlyng Synne 1372-4 ein fast vierthalb hundert jahre früherer beleg zu gewinnen war:

Sawe arwys fro heuene shete And smote men to be dep doun-rygt And one of pe arwys wounded a knygt.

Diese selbe stelle bietet einen beleg für arrow, der zwischen den beiden aus a. 1300 und c. 1386 unter 1303 einzureihen war. Unter hell-fire vermilst man zwischen den a. 1300 und 1526 datierten belegen den $1303 \mathrm{zu}$ datierenden aus R. Brunne's Handlyng Synne 1597-98

pat we be nat with here brent

Yn helle fere (so!) no with here shent. 
Unter know 7 'to have carnal acquaintance or sexual intercourse' fehlt gleich nach dem ersten belege von c. 1200 der aus R. Brunne Handl. Synne 2015-16

Ne pou shalt nat know by wyfe yn holy place, by by lyfe.

Ebenso fehlt unter gleam nach dem 13.. datierten der aus R. Brunne Handl. Synne 225-36

At a wyndowe come yn a beme

And in pe shynynge he saghe a gleme.

Gleicherweise sollte unter glee +2 'musical entertainment' nach dem aus Cursor Mundi 7433 der aus R. Brunne, Handl. Synne $4734 \mathrm{zu}$ finden sein:

bys tolde $y$ for he glemennes sake, To loke whan pey here gle shuld make.

Diese stelle bietet zugleich einen beleg für gleeman, den man unter dem worte ungern vermilst.

Harifford, Conn., U.S. A. Otro B. Schlutter. 